\title{
A dynamical system-Markov model for active postsynaptic responses of muscle spindle afferent nerve
}

\author{
CHEN Xing \& YIN YueHong* \\ State Key Laboratory of Mechanism System and Vibration, Institute of Robotics, Shanghai Jiao Tong University, Shanghai 200240, China
}

Received August 13, 2012; accepted October 15, 2012; published online November 27, 2012

\begin{abstract}
Motoneuron is the control unit of skeletal muscles, and the dynamic frequency-regulating feedback from the afferent nerve of receptors like muscle spindles forms the physical basis of its closed-loop regulation. Focused on the synapses of muscle spindle afferents, this paper established a dynamical system-Markov model starting from presynaptic stimulations to postsynaptic responses, and further verified the model via comparisons between theoretical results and relevant experimental data. With the purpose of describing the active features of dendritic membrane, we employed the methods of dynamical systems rather than the traditional passive cable theory, and identified the physical meaning of parameters involved. For the dynamic behavior of postsynaptic currents, we adopted simplified Markov models so that the analytical solutions for the open dynamics of postsynaptic receptors can be obtained. The model in this paper is capable of simulating the actual non-uniformity of channel density, and is suitable for complex finite element analysis of neurons; thus it facilitates the exploration of the frequency-regulating feedback and control mechanisms of motoneurons.
\end{abstract}

muscle spindle, motoneuron, postsynaptic response, frequency-regulating feedback, synaptic integration

Citation: Chen X, Yin Y H. A dynamical system-Markov model for active postsynaptic responses of muscle spindle afferent nerve. Chin Sci Bull, 2013, 58: 603-612, doi: 10.1007/s11434-012-5562-8

The operation of skeletal muscle is a typically bioelectrochemical closed-loop control process, which is regulated by the action potential (AP) fired by motoneurons. The contraction length/speed and tension of the muscle are fed back by the afferent nerves of muscle spindles and tendon organs within it, and these signals are simultaneously transmitted to the central nervous system (CNS) and the motoneuron itself [1-3]. As an important receptor, the muscle spindle is equivalent to the displacement/speed transducer of skeletal muscle, as the real-time firing rate of its afferent nerves (I a afferents) varies with the length and contraction speed of the muscle. We have proposed the bioelectrochemical frequency-regulating control mechanism for skeletal muscle based on the characteristics of the APs on muscle fibers [4], i.e. the working process of muscle is regulated by the frequency of AP in real time. The afferent signals influence the firing behavior of motoneurons, and thus the feedback of a

*Corresponding author (email: yhyin@sjtu.edu.cn) muscle spindle to the motoneuron forms a local closed loop, which is pivotal to the stability of muscle operation. The researches on the working mechanism of skeletal muscle have lasted for many decades, while the regulation and control mechanisms of muscle are seldom touched; therefore our understanding of the control principium is far from satisfactory. On the other hand, in order to explore the closedloop control properties, the key point is to take extensive researches on the feedback effects of the afferents, and this involves the synaptic transmission between I a afferents and motoneurons.

Motoneurons are myelinated neurons with their dendrites and somas located in the spinal cord [5]. The signals of muscle spindles are excitatory afferents, whose terminals extend into the spinal cord and are connected with the dendritic shafts or spines of motoneurons, and the dendritic tree of one motoneuron can receive thousands of inputs [6]. The investigations on excitatory synapses show that a single input can depolarize the postsynaptic membrane by $0.2-0.4$ 
$\mathrm{mV}$ [7]. Synaptic signals regulate the firing behavior of the axon of a motoneuron via a mechanism called synaptic integration, and the control information is contained in the frequency of AP [4], while because of the "all or none" feature of AP, the quantity regulated by the afferents via synaptic interactions should also be the firing rate of the postsynaptic neuron. Despite of the extensive experimental observations on the process of synaptic integration [8], relevant researches are still at the qualitative stage, and the cybernetic model based on frequency information has not been extracted and proposed. Moreover, the frequencyregulating feedback mechanism of the afferents is closely related to the postsynaptic responses, and the researches on excitatory/inhibitory synapses have been massively carried out [9-11]. Dendrites used to be regarded as purely passive, i.e. postsynaptic currents can only diffuse passively within them. Based on such assumptions, Rall et al. [12] proposed the passive cable theory of membrane, and Hines et al. [13] further developed the NEURON code aimed at calculating the membrane potential responses of the whole neuron. However, recent studies have proved that dendrite structures possess active features, and voltage-sensitive ion channels exist on the dendritic tree [14]. Particularly, the dendritic $\mathrm{Ca}^{2+}$ currents of motoneurons play a key role in their synaptic integration [15]. The passive cable model can hardly simulate the active properties of dendrites, not to say investigating the influence of the non-uniform distribution of channel density on signal processing. For the fact that many difficulties remain in the measurements of the electrical/chemical responses in a single segment of dendrites, theoretical analyses are indispensable for the exploration of the interactions of dendritic information [16], while efficient models being able to easily simulate the active responses of dendrites have not been proposed till today. In effect, the essence of the propagation of membrane potential is the diffusion of cytoplasmic ion; thereby finite element method (FEM) is favorable for the analysis of the non-uniformity of the membrane. Finite element analysis has already been applied in the research of AP propagation on cardiac muscle [17], while for synaptic integration, the main trouble is the high calculation cost of the cable model during the implementation of finite element analysis. It is noteworthy that the dimensionless dynamical system model reflecting the changing characteristics of membrane potential has been proposed [18], and such models can spontaneously simulate the active features of membrane. Besides, based on the chemical kinetics of ion channels, Destexhe et al. $[19,20]$ proposed that postsynaptic responses can be expressed by Markov models. These two models are able to reduce the calculation cost to a large extent; consequently, by means of combining existent research results, this paper aims at establishing a highly efficient computational model that can be used to describe the active postsynaptic responses of Ia afferents, and laying a foundation for the further research of synaptic integration and the frequency-regulating feedback mechanism of motoneurons.

\section{The synapse of I a afferent nerve}

The synapse of I a afferent nerve belongs to chemical type, whose structure is shown in Figure 1. After APs fired by the afferent nerve arrive at presynaptic membrane, the voltage-sensitive $\mathrm{Ca}^{2+}$ channels on which will open when sensing the rise of membrane potential, and presynaptic $\mathrm{Ca}^{2+}$ concentration $\left(\left[\mathrm{Ca}^{2+}\right]\right)$ will increase. The vesicles containing neurotransmitter reside at the presynaptic active zone, and when $\mathrm{Ca}^{2+}$ binds with relevant protein complexes $(\mathrm{x})$, their activated state $\left(\mathrm{x}^{*}\right)$ will be formed. These proteins further combine with the vesicles, causing them to exocytose, and neurotransmitter can be released [21]. For the synapses of I a afferents, the main type of neurotransmitter is glutamate [7], and postsynaptic membrane is densely occupied by ionotropic receptors and metabotropic receptors (also called second-messenger type receptors) that can receive glutamate transmitter. Ionotropic receptor directly opens the ion channel coupled to it after binding with transmitter, and let $\mathrm{Na}^{+}, \mathrm{K}^{+}$and $\mathrm{Ca}^{2+}$ pass through the membrane, thus it dominates the rapid transmission of synaptic signal. Metabotropic receptor mainly contributes to the slow and long term regulation of postsynaptic responses, while for the feedback activities of motoneurons during normal operation of muscle, the instantaneous dynamic response is more significant, so this paper only deals with ionotropic receptors. On the other hand, ionotropic receptors can be classified into two categories according to the types of transmitter that they receive [7]: one category is called $N$-methyl-D-aspartate (NMDA) receptor, i.e. this kind of receptors bind with NMDA-type transmitter to open, and the other class is non-NMDA receptor. These two types of receptors coexist in the synapses of motoneurons; therefore the excitatory postsynaptic current during rapid signaling is the superposition of their respective currents. It should be noted that the exocytosis of vesicles requires the increase of presynaptic

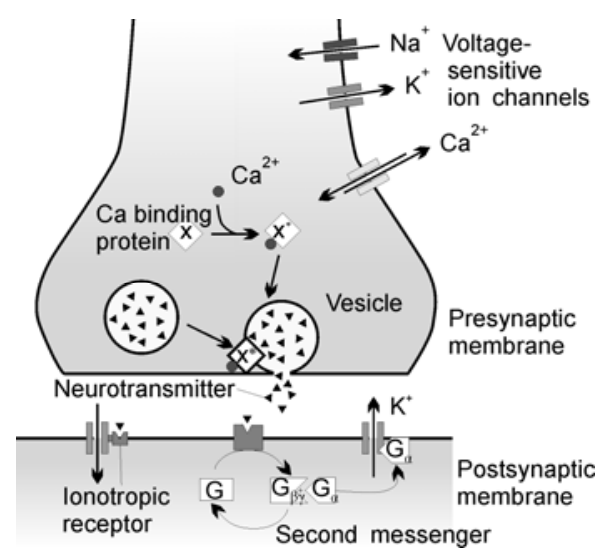

Figure 1 The structure of chemical synapses. 
$\left[\mathrm{Ca}^{2+}\right]$, which is caused by presynaptic depolarization. We see that for chemical synapses, there always exists a cycle in which presynaptic potential induces postsynaptic current, which is transferred into presynaptic potential again, and thus, it is necessary to build a dynamic model for membrane potential first.

\section{The dynamical system model for membrane potential}

Since the generation mechanism of AP was discovered, several kinds of mathematical models describing the dynamical features of membrane potential have been proposed. The earliest systematic and quantitative researches on AP were conducted by Hodgkin and Huxley [22], who concluded the coupling relationships among membrane potential, channel conductance and time based on the chemical characteristics of ion channels, and the resultant model was named as the $\mathrm{H}-\mathrm{H}$ model. This model succeeded in the dynamic description of membrane potential, however, the $\mathrm{H}-\mathrm{H}$ model includes 4 state variables, and the computation involves 4 coupled differential equations. The passive cable model is similar to the $\mathrm{H}-\mathrm{H}$ model for that it belongs to electrical models as well. Although the variables in the cable model is fewer, solving temporal and spatial coupled parabolic partial differential equations is needed [12]; as a result, electrical models are not suitable for the calculation of complex activities of neurons. As previously mentioned, in order to elaborately analyze the mechanisms of synaptic integration, a model aimed at complicated finite element computation should be proposed. FitzHugh [18] proposed a dynamical system model (the FitzHugh-Nagumo model) composed of two differential equations, which can both represent the main time-domain features of AP and considerably reduce the complexity of computation. Besides, Hindmarsh and Rose [23] corrected the defect that the FitzHugh-Nagumo model is not capable of reasonably reflecting the relation between AP frequency and control current ( $f-I$ relation). Another advantage of dynamical system models is that computation can be carried out dimensionlessly, degrading the requirements on temporal and spatial iteration steps. This paper adopts the structure of the Hindmarsh-Rose model to implement the real-time calculation of membrane potential, and the model can be described by two coupled differential equations:

$$
\left\{\begin{array}{l}
\dot{x}=-a[f(x)-y-z], \\
\dot{y}=b[f(x)-q \exp (r x)+s-y],
\end{array}\right.
$$

where $f(x)=c x^{3}+d x^{2}+e x+h$. The membrane potential is denoted by $x$, and $y$ represents the natural current across the membrane, $z$ can be regarded as the stimulation current (injection current), while other parameters are all constant. If setting the two equalities in eq. (1) to zero, the fixed point of the system corresponding to the resting state of membrane can be obtained. Although the calculation of eq. (1) can be dimensionless, and the fixed point can be freely chosen from the perspective of mathematics, here we let $y$ and $z$ be zero and $x$ be negative when the system is at equilibrium (with no stimulation current and net membrane current, resting potential negative), so that the system is consistent with the actual physiological features of membrane. Thus, at equilibrium, the system can be written as

$$
\left\{\begin{array}{l}
f\left(x_{\text {ep }}\right)=0, \\
-q \exp \left(r x_{\text {ep }}\right)+s=0,
\end{array}\right.
$$

where $x_{\mathrm{ep}}$ is the $x$-coordinate of the fixed point, so we have

$$
x_{\mathrm{ep}}=\frac{1}{r} \ln \left(\frac{s}{q}\right) \text {. }
$$

In order to assure the resting potential being negative, there must be $s<q$. The parameters of the two equalities in eq. (2) can be decided independently, and finally we chose the dimensionless resting potential as about -110 , i.e. the fixed point is $(-110,0)$, and the parameters of the system in this case are listed in Table 1. If we iterate the system with the 4th order Runge-Kutta method, the phase portrait of the system $(z=10)$ can be drawn as shown in Figure 2(a), in which the dashed lines characterize the $x$ - and $y$-nullclines. The state of the system will go through a periodic orbit when it deviates from the fixed point, and this corresponds to the reciprocating change of the membrane potential when AP train occurs (Figure 2(b)). Furthermore, it can be noted from eq. (1) that the parameters $a$ and $b$ determine the changing rates of $x$ and $y$, respectively. Due to the rapid change of $x$ and the slower change of $y$, the system is very sensitive to $b$. We see from the second equality of eq. (1) that the parameter $b$ actually decides the changing rate of membrane currents, and thereby $b$ can be considered as corresponding to the densities of $\mathrm{Na} / \mathrm{K} / \mathrm{Ca}$ channels on the membrane, i.e. under the same injection current, the firing rate of AP should be positively correlated to the value of $b$. Figure 3(a) shows the frequencies of AP when $b$ is 30, 60 and 90, respectively, with the same injection current $(z=10)$. It must be noted that if $b$ is simply fixed, we are actually adjusting the densities of all the types of channels simultaneously, while during the practical application of the model, sometimes only one of these densities need to be changed.

Table 1 Parameters in the dynamical system model of the membrane

\begin{tabular}{cc||cc}
\hline Parameter & Value & Parameter & Value \\
\hline$a$ & $4 \times 10^{3}$ & $h$ & -14.297 \\
$b$ & 30 & $q$ & $1.464 \times 10^{3}$ \\
$c$ & $1.7 \times 10^{-4}$ & $r$ & $1 \times 10^{-1}$ \\
$d$ & $2 \times 10^{-2}$ & $s$ & $2.4 \times 10^{-2}$ \\
$e$ & $1 \times 10^{-2}$ & & \\
\hline
\end{tabular}



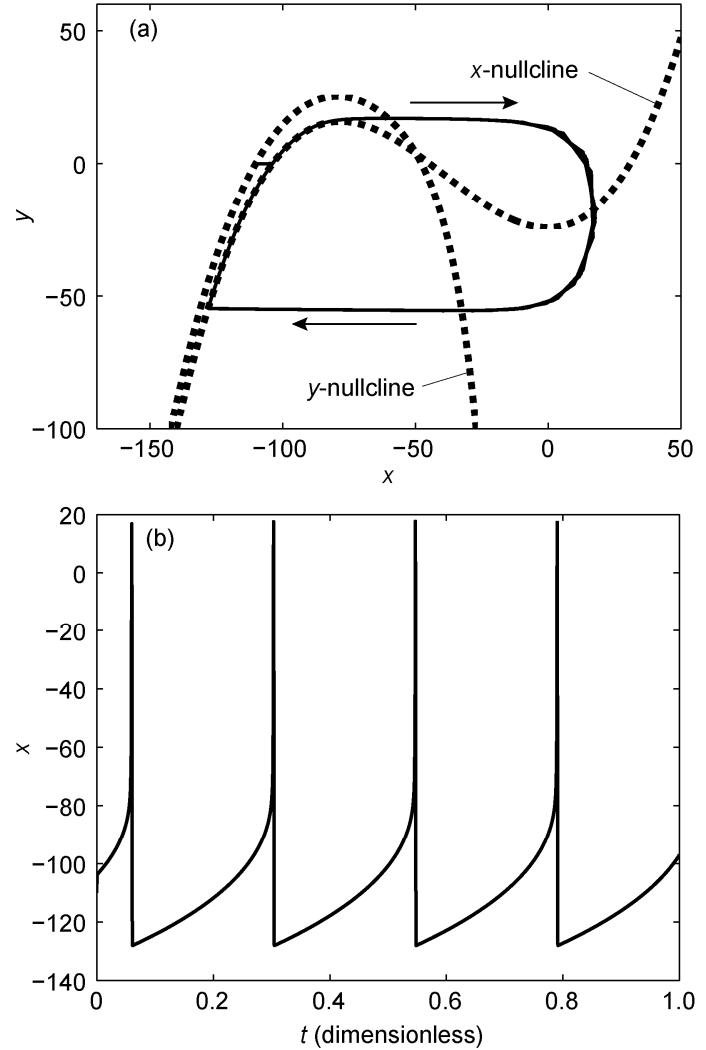

Figure 2 (a) The phase portrait of the dynamical system; (b) the time-domain feature of variable $x$.

For a certain membrane domain, the number of $\mathrm{K}^{+}$channels relative to that of $\mathrm{Na}^{+} / \mathrm{Ca}^{+}$channels determines the amplitude, duration and frequency of AP [24], therefore the parameter characterizing the density of $\mathrm{K}^{+}$channels needs to be separated. As for the total membrane current, $\mathrm{Na}^{+}$channels and $\mathrm{Ca}^{+}$channels mainly contribute to the rising phase of the current, while its falling phase attributes to the effect of $\mathrm{K}^{+}$ channels [22], so when $\dot{y} \geqslant 0, f(x)-q \cdot \exp (r x)+s \geqslant y$.

We see that the value of $y$ is under its nullcline when the current is increasing. The value of $b$ in this case corresponds to the densities of $\mathrm{Na}^{+} / \mathrm{Ca}^{+}$channels, and we denote it as $b_{1}$. When $\dot{y}<0, f(x)-q \cdot \exp (r x)+s<y$.

This implies when the current is decreasing, $y$ is above its own nullcline, and now $b$ corresponds to the density of $\mathrm{K}^{+}$ channels, which is denoted by $b_{2}$. Hence, the non-uniform distribution of channel densities can be simulated via adjusting the values of $b_{1}$ and $b_{2}$ in different domains of the membrane. Furthermore, if defining $\lambda=b_{1} / b_{2}$, the density ratio of $\mathrm{Na} / \mathrm{K}$ channels is represented by $\lambda$. We take $b_{1}=30$ and $z=12$, and then Figure 3(b) shows the comparisons of the time-domain properties of a single AP and the frequencies of AP trains when $\lambda=1$ and $\lambda=30$, respectively. It can be checked that with the increase of $\lambda$, the amplitude, duration, as well as the frequency of AP will all grow in response, and this feature coincides with the electrophysiological principles of membrane.

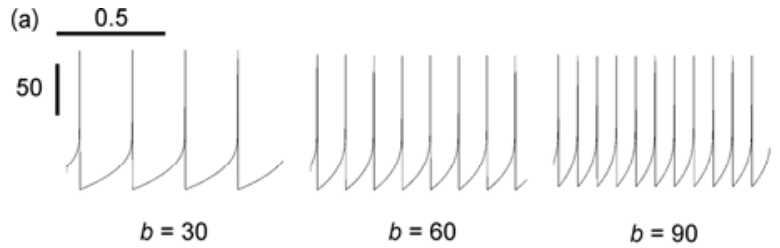

(b)
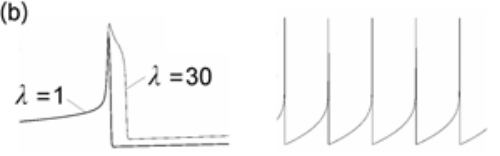

$\lambda=1$

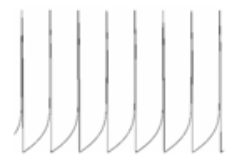

$\lambda=30$

Figure 3 (a) The effect of $b$ on the frequency of AP; (b) the effect of $\lambda$ on the features of AP.

Identifying the physical meaning of $b$ is significant to the research on the frequency-regulating feedback mechanism of neurons, for the reason that the channel densities at different locations of a neuron may differ a lot, forming the unique function of information modulation [24]. In the rest part of this paper, if not particularly specified, the parameter $b$ corresponds to the situation where $\lambda=1$. One extra thing that is worth noting is that the dynamical system model represented by eq. (1) characterizes the response of a closed membrane domain to the injection current, while for an open domain, the diffusion effect of the charges inside the membrane should be considered.

On the other hand, according to Figure 2(b), when AP is induced, the peak value of $x$ is about 18 , corresponding to the real spike of AP $(40 \mathrm{mV})$, while at equilibrium the value of $x(-110)$ corresponds to the resting potential (-65 $\mathrm{mV}$ ), and therefore the transformation between the dimensionless voltage $V_{\mathrm{dim}}$ and the real voltage $V_{\mathrm{mV}}$ in the unit of $\mathrm{mV}$ can be obtained as

$$
k \cdot V_{\mathrm{dim}}+\Delta=V_{\mathrm{mV}},
$$

where the ratio constant $k=0.82$, and $\Delta=25.24$. In the same way, if we match the dimensionless time with physical time, the model can be applied to the firing behavior analysis of neurons in different frequency domains.

\section{The kinetic model for postsynaptic receptors}

As shown in Figure 1, the open of the ionotropic receptors on the postsynaptic membrane requires their binding with corresponding transmitter, and the receptors will experience the states of close and/or desensitization. The transition rates between these states may depend on the surrounding transmitter concentration. Markov models can be used to describe chemical kinetic systems whose transition rates do not vary with time, and relevant studies [25] have shown that, Markov models can provide reasonable descriptions for ligand-gated ion channels like postsynaptic receptors. Besides, the open fraction of the receptors is directly related 
to the transmitter concentration in the synaptic gap, while the release quantity of transmitter is determined by presynaptic $\left[\mathrm{Ca}^{2+}\right]$, so if we build the model according to the physiological open process of the receptors, the relations between presynaptic $\left[\mathrm{Ca}^{2+}\right]$ and ion currents, the dynamic recycling process of $\mathrm{Ca}^{2+}$ by ionic pumps, the activation process of $\mathrm{Ca}^{2+}$ binding protein complex, as well as the releasing and recycling kinetics of transmitter [20] should all be taken into account. In this way too many state variables and differential equations are involved; thus, it is very difficult to get a kinetic model of receptors for complex computation and whole cell simulation from the perspective of detailed physiology. There has been detailed modeling for postsynaptic responses via Markov models with 6 state variables to describe the open fraction of receptors [26]; however, Destexhe et al. [20] found that a 3-state Markov model is enough to grasp the main features of the open kinetics via comparisons between computational results and experimental data, and the dependence of transition rates on transmitter concentrations can be simplified into a piecewise pulse function. Therefore this paper describes the open kinetics of receptors with 3-state models.

\subsection{Non-NMDA receptors}

The 3-state Markov model for non-NMDA receptors is shown in Figure 4, where C, O and D characterize the states of close, open and desensitization respectively, and the transition rates are denoted by $r_{i}(i=1,2,3,5)$, in which $r_{1}$ is the function of transmitter concentration [T]. We assume that $[\mathrm{T}]$ is a pulse function with the pulse width of $1 \mathrm{~ms}$. When an AP train occurs at the presynaptic membrane, if the membrane potential exceeds $0 \mathrm{mV}$ at the rising phase of $\mathrm{AP},[\mathrm{T}]=1 \mathrm{mM}$, and it returns to zero after $1 \mathrm{~ms}$ [20]. Thus $r_{1}$ is a piecewise function with each segment constant, and the advantage is that the analytical solutions of the timevarying open fraction of receptors can be obtained.

The presynaptic AP train can be generated by the dynamical system model illustrated in eq. (1). Let the dimensionless time 0.004 correspond to $1 \mathrm{~ms}$, then we can calibrate that the dimensionless injection current needed for producing an AP train of $20 \mathrm{~Hz}$ is $z=12$, and the real stimulus is about $0.1 \mathrm{nA}$ according to experimental data [26]. So the relation between the dimensionless time $t_{\mathrm{dim}}$ and the physical time $t_{\text {real }}$ in the unit of $\mathrm{s}$ is

$$
k_{\mathrm{t}} \cdot t_{\text {dim }}=t_{\text {real }},
$$

where $k_{\mathrm{t}}=0.25$. The relation between the dimensionless

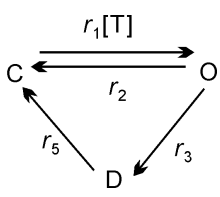

Figure 4 The Markov model for non-NMDA receptors. current $I_{\mathrm{dim}}$ and the real current $I_{\text {real }}$ in the unit of nA can be listed as

$$
k_{\mathrm{I}} \cdot I_{\text {dim }}=I_{\text {real }},
$$

where $k_{\mathrm{I}}=8.33 \times 10^{-3}$. When $[\mathrm{T}]=1 \mathrm{mM}$, the solution of the Markov model in Figure 4 can be written as

$$
\left\{\begin{aligned}
O\left(t-t_{0}\right)= & O_{\infty}+K_{1 h} \exp \left[-\left(t-t_{0}\right) / \tau_{1}\right] \\
& +K_{2 h} \exp \left[-\left(t-t_{0}\right) / \tau_{2}\right] \\
D\left(t-t_{0}\right)= & D_{\infty}+K_{3 h} \exp \left[-\left(t-t_{0}\right) / \tau_{1}\right] \\
& +K_{4 h} \exp \left[-\left(t-t_{0}\right) / \tau_{2}\right]
\end{aligned}\right.
$$

where $O$ denotes the open fraction, and $D$ is the desensitization fraction. The time when [T] changes is denoted by $t_{0}$, and the parameters involved are as follows:

$$
\begin{aligned}
O_{\infty} & =\frac{-\delta r_{1}}{\alpha \delta-\beta \gamma}, \\
K_{1 h} & =\frac{\left(O_{0}-O_{\infty}\right)\left(\alpha+\tau_{2}^{-1}\right)+\beta\left(D_{0}-D_{\infty}\right)}{\tau_{2}^{-1}-\tau_{1}^{-1}}, \\
K_{2 h} & =\left(O_{0}-O_{\infty}\right)-K_{1 h}, \\
z_{\infty} & =\frac{\gamma r_{1}}{\alpha \delta-\beta \gamma}, \\
K_{3 h} & =K_{1 h} \frac{-\alpha-\tau_{1}^{-1}}{\beta}, \\
K_{4 h} & =K_{2 h} \frac{-\alpha-\tau_{2}^{-1}}{\beta}, \\
\tau_{1,2}{ }^{-1} & =-\frac{\alpha+\delta}{2} \pm \frac{1}{2} \sqrt{(\alpha-\delta)^{2}+4 \beta \gamma},
\end{aligned}
$$

where $O_{0}$ and $D_{0}$ are the initial values of the open fraction and desensitization fraction, and we have $\alpha=-r_{1}-r_{2}-r_{3}, \beta=$ $-r_{1}, \gamma=r_{3}$, and $\delta=-r_{5}$. When [T] $=0$, the solution is

$$
\begin{aligned}
& \left\{\begin{array}{l}
O\left(t-t_{0}\right)=K_{1 l} \exp \left[-\left(t-t_{0}\right) / \tau_{3}\right], \\
D\left(t-t_{0}\right)=K_{2 l} \exp \left[-\left(t-t_{0}\right) / \tau_{3}\right]+K_{3 l} \exp \left[-\left(t-t_{0}\right) / \tau_{4}\right]
\end{array}\right. \\
& K_{1 l}=O_{0}, \\
& K_{2 l}=\frac{\gamma K_{1 l}}{-\delta-\tau_{3}^{-1}}, \\
& K_{3 l}=D_{0}-K_{2 l}, \\
& \tau_{3}^{-1}=\beta-\alpha, \\
& \tau_{4}^{-1}=-\delta \text {. }
\end{aligned}
$$

By adjusting the transition rates between states in the model, it can be applied to the descriptions of the open kinetics of different non-NMDA receptors.

\subsection{NMDA receptors}

The activation properties of NMDA receptors are distinct 
from that of non-NMDA ones, because their open and desensitization rates are both slower. The corresponding Markov model is shown in Figure 5, from which we see that for NMDA receptors, the open state can only turn into the closed state instead of the desensitization state, which, on the contrary, can be transferred into both the open and closed states. The transition rates are still $r_{i}(i=2,4,5,6)$, in which $r_{6}$ depends on [T], which is the same pulse function defined above. When $[\mathrm{T}]=1 \mathrm{mM}$, the form of the solution of the Markov model in Figure 5 is the same as that presented in eq. (7), except that $O_{\infty}$ and $D_{\infty}$ become

$$
\begin{aligned}
& O_{\infty}=\frac{\beta r_{6}}{\alpha \delta-\beta \gamma}, \\
& z_{\infty}=\frac{-\alpha r_{6}}{\alpha \delta-\beta \gamma},
\end{aligned}
$$

where $\alpha=-r_{2}, \beta=r_{4}, \gamma=-r_{6}$, and $\delta=-r_{4}-r_{5}-r_{6}$. When [T] $=0$, the solution becomes

$$
\left\{\begin{array}{l}
O\left(t-t_{0}\right)=K_{1 l} \exp \left[-\left(t-t_{0}\right) / \tau_{3}\right]+K_{2 l} \exp \left[-\left(t-t_{0}\right) / \tau_{4}\right] \\
D\left(t-t_{0}\right)=K_{3 l} \exp \left[-\left(t-t_{0}\right) / \tau_{3}\right]
\end{array}\right.
$$

$$
\begin{gathered}
K_{1 l}=\frac{\beta K_{3 l}}{-\alpha-\tau_{3}^{-1}}, \\
K_{2 l}=O_{0}-K_{1 l}, \\
K_{3 l}=D_{0}, \\
\tau_{3}^{-1}=\gamma-\delta, \\
\tau_{4}^{-1}=-\alpha .
\end{gathered}
$$

\section{The model for postsynaptic responses}

The open kinetics of non-NMDA and NMDA receptors directly influence the postsynaptic responses, i.e. the excitatory postsynaptic current (EPSC) and the excitatory postsynaptic potential (EPSP). For the postsynaptic neuron, EPSC is equivalent to the injection current $z$ in eq. (1), and EPSP corresponds to $x$. With a certain total conductance of postsynaptic receptors, the instantaneous value of EPSC is decided by the present EPSP based on the Ohm's law. However, according to eq. (1), EPSP is also dynamically influenced by EPSC, so these two quantities need to be iterated in a coupled way during computation. On the other hand, based on the superposition principle of currents, EPSC is composed of the currents of non-NMDA and NMDA receptors, and remarkable differences exist in their magnitudes and modulation factors.

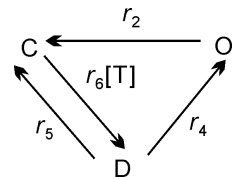

Figure 5 The Markov model of NMDA receptors.

\subsection{EPSC}

(i) The current of non-NMDA receptors. At physiological conditions, the behavior of non-NMDA receptors can only be modulated by transmitter concentration; thereby the EPSC can be calculated as

$$
I_{\mathrm{n}}=\bar{g}_{\mathrm{n}} \cdot O_{\mathrm{n}} \cdot\left(E-E_{\mathrm{rev}}\right),
$$

where $I_{\mathrm{n}}$ is the non-NMDA current, and $\bar{g}_{\mathrm{n}}$ is the maximum conductance of receptors. From the physiological data [7], the single channel conductance of non-NMDA receptors is far less than $20 \mathrm{pS}$, while the maximum postsynaptic conductance is about $\bar{g}_{\mathrm{n}}=0.4 \mathrm{nS}$. The open fraction is represented by $O_{\mathrm{n}}, E$ is the postsynaptic potential, and the reversal potential (equilibrium potential) is denoted by $E_{\mathrm{rev}}$. For glutamate ionotropic receptors, $E_{\mathrm{rev}}=0$.

Non-NMDA current is dominant in EPSC [27], especially at the initial increasing stage of the current. However, non-NMDA receptors desensitize rapidly, and thus their currents decay very fast under the repetitive stimulations of the AP train. In order to match the non-NMDA current generated by the model with experimental results, the transition rates of the model shown in Figure 4 are chosen as listed in Table 2. Assume that the frequency of the presynaptic AP train is $20 \mathrm{~Hz}$, then its waveform can be calculated with eq. (1) (Figure 6(a)), and the pulse train of [T] generated by the model is shown in Figure 6(b). The response of non-NMDA current can be further obtained with eqs. (7) and (8) (Figure $6(c)$, thin solid line). Note that the excitatory current (inward current) is defined negative. Figure 6(c) shows that the amplitudes of the current as well as the open fraction are higher under the stimulations of the first 2-3 pulses, while they decay rapidly and approach the steady state. This result is consistent with the relevant voltage-clamp experiments on postsynaptic membrane [20].

(ii) The current of NMDA receptors. The modulation process of NMDA receptors is much more complicated than that of non-NMDA receptors, for that their open process is affected by 3 factors: the transmitter concentration, postsynaptic potential and extracellular $\left[\mathrm{Mg}^{2+}\right]$. When the membrane is at the vicinity of the resting potential, the

Table 2 The transition rates in the Markov models of non-NMDA and NMDA receptors

\begin{tabular}{lccccc}
\hline Receptor type & $r_{1}\left(\mathrm{~s}^{-1} \mathrm{mM}^{-1}\right)$ & $r_{2}\left(\mathrm{~s}^{-1}\right)$ & $r_{3}\left(\mathrm{~s}^{-1}\right)$ & $r_{4}\left(\mathrm{~s}^{-1}\right)$ & $r_{5}\left(\mathrm{~s}^{-1}\right)$ \\
\hline Non-NMDA & 1000 & 10 & 50 & - & 2 \\
NMDA & - & 6.9 & - & 160 & 4.7 \\
\hline
\end{tabular}


pores of the ion channels of NMDA receptors are occupied by $\mathrm{Mg}^{2+}$ [7]; consequently, even if the receptors are bound with neurotransmitter, the channels remain closed. These channels can be opened only if the depolarization of the postsynaptic membrane occurs, and $\mathrm{Mg}^{2+}$ leaves the pores under the effect of electromotive force across the membrane. Thus, when calculating NMDA current, a function related to membrane potential and extracellular $\left[\mathrm{Mg}^{2+}\right]$ needs to be introduced [28]:

$$
G=\frac{1}{1+\left([\mathrm{Mg}]_{\mathrm{o}} / 3.57\right) \exp (-0.062 E)},
$$

where $[\mathrm{Mg}]_{0}$ denotes the extracellular $\left[\mathrm{Mg}^{2+}\right]$, which can be regarded as constant at physiological state: $[\mathrm{Mg}]_{\mathrm{o}}=1 \mathrm{mM}$. $E$ is the membrane potential in $\mathrm{mV}$, so the NMDA current $I_{\mathrm{N}}$ can be written as

$$
I_{\mathrm{N}}=\bar{g}_{\mathrm{N}} \cdot G \cdot O_{\mathrm{N}} \cdot\left(E-E_{\mathrm{rev}}\right),
$$

where the meaning of $E$ stays the same, and the reversal potential $E_{\text {rev }}=0$. The maximum conductance of NMDA receptors is denoted by $\bar{g}_{\mathrm{N}}$, and the single channel conductance is about $50 \mathrm{pS}$ [7]. Compared with non-NMDA receptors, the conductivity of NMDA receptors is larger, because they allow $\mathrm{Ca}^{2+}$ to pass besides $\mathrm{Na}^{+}$and $\mathrm{K}^{+}$. However, experiments show that the contribution of NMDA current to EPSC is very small [27], and it only takes effect during the late components of EPSC for rapid signaling. Therefore, the number of NMDA receptors on the postsynaptic membrane is much smaller than that of non-NMDA ones. If setting their density ratio per unit area to be $1: 100$, we have $\bar{g}_{\mathrm{N}}=0.5 \mathrm{nS}$. In the CNS, the main function of NMDA receptors is to generate long-term potentiation (LTP) effect [29], while for I a afferents of muscle spindles, our purpose is to investigate the fast dynamic responses, i.e. the LTP effect will not be considered in this paper.
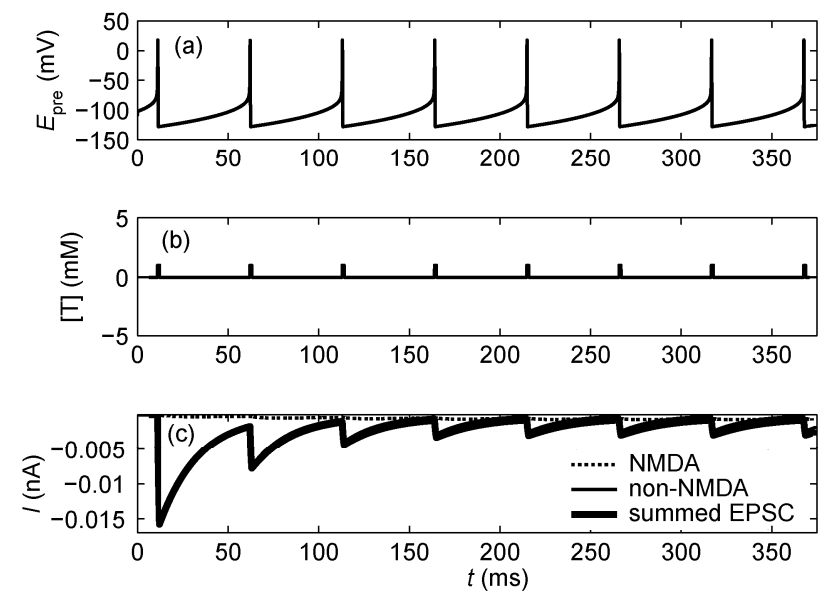

Figure 6 (a) Presynaptic AP train; (b) transmitter concentration [T]; (c) the summed EPSC, NMDA and non-NMDA currents.
The transition rates involved in the model of NMDA receptors are also listed in Table 2. The presynaptic stimulus is the same as previously described, and the NMDA current can be computed with eqs. (7) and (9), as shown in Figure 6(c) (dashed line). The total EPSC is formed by the summation of the non-NMDA and NMDA currents (Figure 6(c), bold solid line), from which we see that EPSC faithfully reserves the frequency information of presynaptic AP. If comparing the total current with the NMDA current, it is easy to find that at the rising stage of the current, NMDA current imposes little effect, while at the falling phase, it contributes to the tail current of EPSC. Moreover, after repetitive stimulations, NMDA current gradually becomes remarkable and stable, consistent with experimental phenomena [27].

\subsection{EPSP}

EPSP refers to the depolarization responses of dendrites or the soma under the effect of EPSC. As mentioned above, due to the active properties of dendrites, EPSP can differ a lot from that in passive conditions where EPSP rapidly attenuate with the increasing distance from the synapse, while for active dendrites, the amplitude of EPSP can keep constant or even be amplified [14] during its propagation. The dynamical system model can conveniently simulate the active features of the membrane, so if we take EPSC as the injection current $z$, the model represented by eq. (1) can still be applied to the calculation of EPSP. For the fact that a single excitatory synapse can only depolarize the postsynaptic membrane by less than $1 \mathrm{mV}$, and compared with the axon, the $\mathrm{Na} / \mathrm{K}$ channel densities of the postsynaptic membrane are 40 folds lower [24], as a result, the parameter $b$ needs to be re-adjusted so as to reflect the actual physiological characteristics of the membrane, and we take $b=30 / 40$ $=0.75$. On the other hand, Figure 6(c) shows that after repetitive stimulations by the presynaptic AP train, EPSC becomes stable, and this is equivalent to a constant average injection current $I_{\mathrm{i}}$. As discussed previously, eq. (1) represents the response of a closed membrane domain to the injection current; therefore at the steady state, the variation of the charge inside postsynaptic membrane can be denoted as

$$
\bar{Q}=I_{\mathrm{i}} t+Q_{0},
$$

where $Q_{0}$ is the initial charge. The instantaneous membrane potential is proportional to the difference of the positive charge densities across the membrane [4], so the mean postsynaptic potential $\bar{U}$ can be expressed as

$$
\bar{U}=k_{\mathrm{c}}\left(\bar{Q} / V-\rho_{0}\right)=\left(k_{\mathrm{c}} / V\right) I_{\mathrm{i}} t+C_{0},
$$

where $k_{\mathrm{c}}$ is a constant ratio, $V$ is the effective volume of the closed membrane domain, and the extracellular charge density is denoted by $\rho_{0}$, which can be assumed constant. $C_{0}$ 
is also a constant, and $C_{0}=\left(k_{\mathrm{c}} / V\right) Q_{0}-k_{\mathrm{c}} \rho_{0}$. Eq. (14) shows that when EPSC reaches the steady state, EPSP will linearly grow with time due to charge accumulation. However, under actual physiological conditions, the charge injected into the postsynaptic membrane will diffuse to farther regions, and when the steady state is achieved, there should be no net current in the finite volume of postsynaptic region, i.e. the equilibrium between the injected current and diffusing current is reached. Thus, when computing EPSP, the steady state mean current $I_{\mathrm{i}}$ needs to be removed so as to match with the real situation. The variation of EPSP can be obtained by substituting the resultant EPSC into eq. (1), as shown in Figure 7. Figure 7(a) and (b) separately show the comparisons between the computed EPSP and experimental results [30] under the presynaptic stimulus of $5 \mathrm{~Hz}$ and 40 Hz. It should be noted that the experimental EPSP in Figure 7 was measured at the soma, and the amplitude attained several milli-volts, which implied that EPSP was amplified during its propagation from dendrites to the soma [31]. In order to better verify the model, we magnify the theoretical EPSP by the same extent, and it is clear that the dynamic features presented by the simulation results are consistent with that of actual cases. Furthermore, experimental researches have revealed that the decay rate of EPSP under high-frequency stimulus is larger than that stimulated by lower frequency [30], and by comparing the results of $5 \mathrm{~Hz}$ and $40 \mathrm{~Hz}$, we see that our model faithfully represented such dynamical features as well.

On the other hand, relevant researches show that the steady state amplitudes of EPSP (like the last two EPSP spikes in Figure 7(a)) depend on the frequency of presynaptic stimulus, i.e. the higher the frequency, the lower the steady state amplitude, which decays exponentially as the general trend, and this effect is called the redistribution of synaptic efficacy [30]. For the model in this paper, if we investigate the EPSP under different stimulus frequencies and denote the steady state EPSP as EPSP st $_{\text {t }}$ the relation between the frequency $f$ and the $\operatorname{EPSP}_{\text {st }}$ can be obtained as shown in Figure 8(a), in which the data can indeed be expressed by an exponential function, and the trend is the same as that of experimental results. Thus the effectiveness of the model has been further verified, and the fit function for the curve in Figure 8(a) is

$$
\mathrm{EPSP}_{\mathrm{st}}=c_{1} \exp \left(-c_{2} f\right)+c_{3},
$$

where $c_{1}=3.376, c_{2}=0.152, c_{3}=-64.36$. Figure 8(b) shows the variation trend of EPSP st with $\lambda$ when $b_{1}$ and $b_{2}$ are respectively fixed at 0.75 , under the presynaptic stimulus of $20 \mathrm{~Hz}$. We see that EPSP $_{\mathrm{st}}$ almost does not vary with $\lambda$ when $b_{1}$ is fixed, while when $b_{2}$ is fixed, EPSP $\mathrm{st}_{\text {st }}$ approximately grows linearly with the increasing $\lambda$. As previously discussed, for the reason that $b_{1}$ mainly characterizes the density of $\mathrm{Na}^{+}$channel, and $b_{2}$ represents the density of $\mathrm{K}^{+}$ channel, therefore under the stimulation of constant frequency, the amplitude of EPSP $\mathrm{st}_{\text {st }}$ is dominated by the value of $b_{1}$, while $b_{2}$ is mainly responsible for the kinetics of the decreasing phase of EPSP. In other words, different changing modes of $\lambda$ will lead to distinct influences on $\mathrm{EPSP}_{\mathrm{st}}$, and this is consistent with the principles of depolarization response of the membrane. (a)
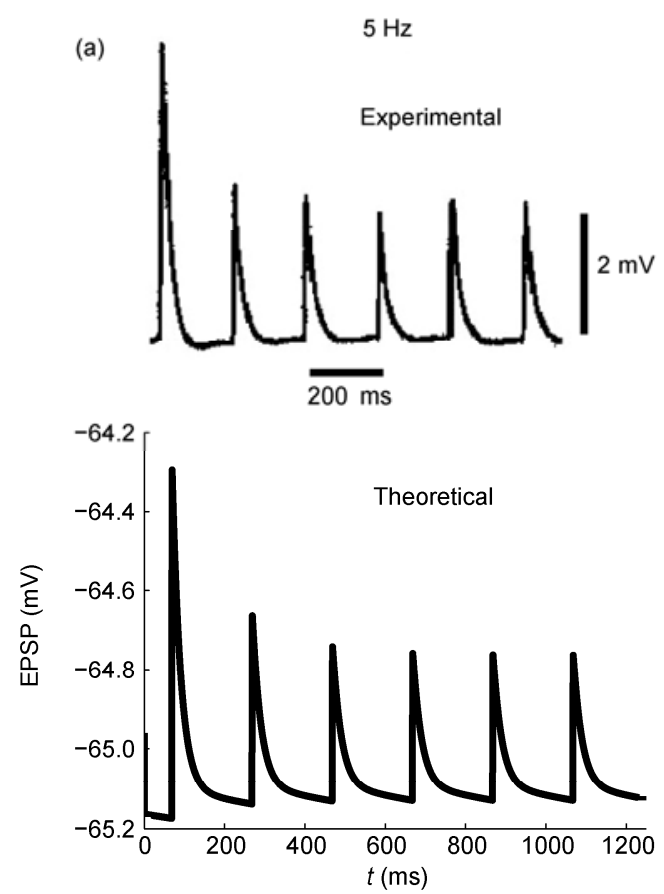

(b)
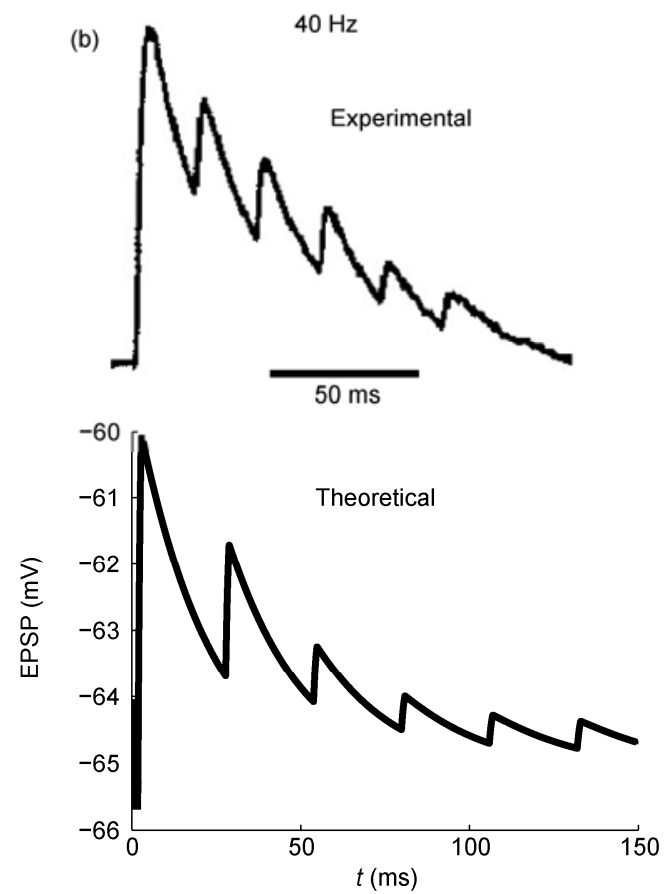

Figure 7 The comparisons between theoretical and experimental EPSP. (a) $5 \mathrm{~Hz}$; (b) $40 \mathrm{~Hz}$. 

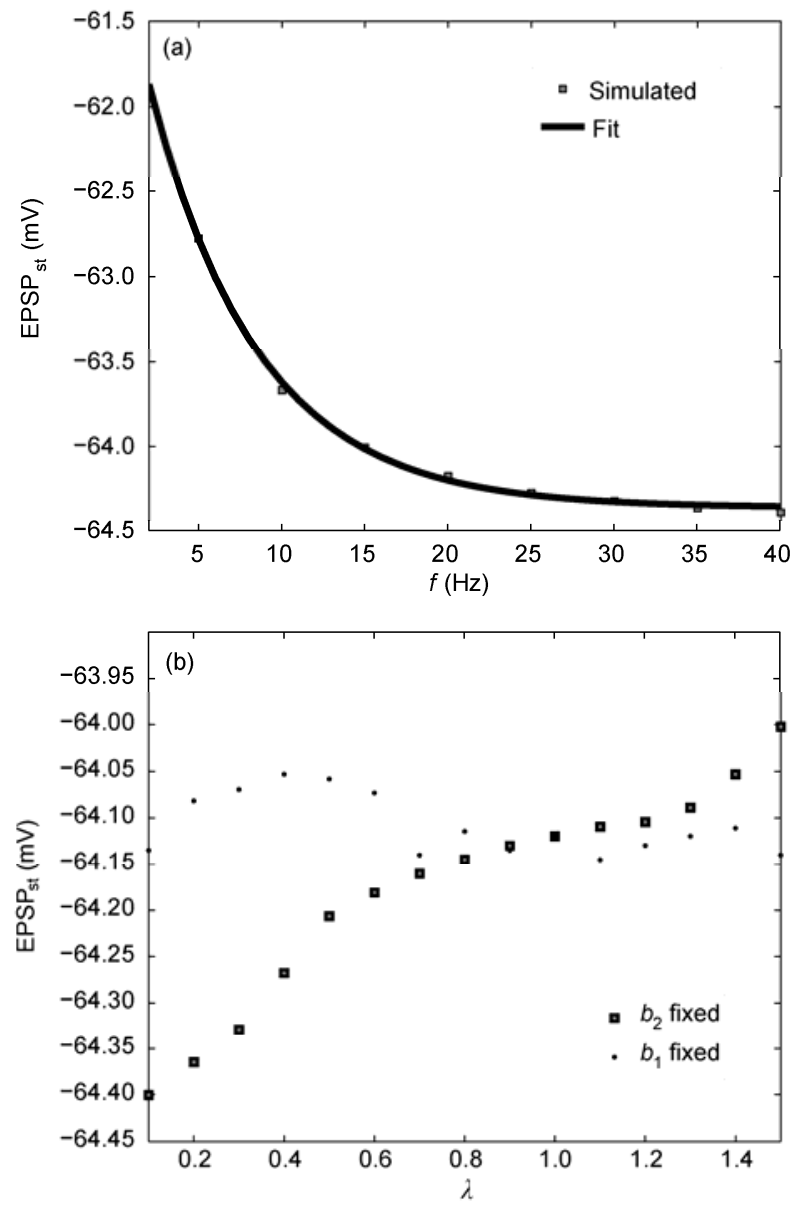

Figure 8 (a) The relation between $\mathrm{EPSP}_{\mathrm{st}}$ and $f$; (b) the relation between EPSP $_{\mathrm{st}}$ and $\lambda$ when $b_{1}$ and $b_{2}$ are fixed respectively.

\section{Discussion}

By introducing the dynamical system modeling method for membrane potential and the Markov model for ionotropic receptors, this paper established a highly efficient computational model for the active postsynaptic responses (EPSC, EPSP) of I a afferents of muscle spindles with the combination of the advantages of these two methods. The correctness and effectiveness of the model has been validated via comparisons with relevant experimental data. Different from the traditional passive cable theory, this model does not simply treat the membrane as a coupled structure of capacitors and resistors; instead, based on the intrinsic properties of the dynamical system, the model calculates the dynamic responses of the potential of a membrane region under the effect of the stimulating current. Particularly, we identified the physical meaning of relevant parameters $(b, \lambda)$, making it possible to independently adjust the physiological characteristics (the densities of $\mathrm{Na} / \mathrm{K} / \mathrm{Ca}$ channels) of a certain membrane domain with these parameters, which enable the model to simulate the active electrophysiological features of non-uniform membrane. Although this paper only involved the simulation of the potential in a closed region, actually if we express the injection current $(z)$ as a diffusion item, the spread of the current and the interactions of the potential among different membrane domains can be computed. Thereby, the modeling method we proposed can be easily applied in the finite element analysis of motoneurons, and the disadvantage of the electrical model which cannot reflect the detailed features of the membrane can be overcome. For postsynaptic responses, we employed the simplified Markov model, which covered the main features of EPSC, and at the same time avoided introducing too many differential equations and state variables, so that the computation cost can be greatly saved. When calculating EPSP, we combined the Markov model with the dynamical system model to make the model capable of simulating the active propagation property of the postsynaptic membrane. Thus, from the generation of presynaptic AP train to the response of EPSP, we need only to solve twice the first order system of differential equations (eq. (1)), and EPSC can be obtained directly from the analytical expressions (eqs. (7)-(9)). The model can be applied to the real-time computation of synaptic responses, i.e. we can get the postsynaptic response under the presynaptic stimulus of dynamically varying frequency. Moreover, despite that the calculations in this paper are transferred into dimensional form so as to compare with the experimental data, the final purpose is to extract essential mathematical rules of synaptic integration and information processing during actual complicated computations; therefore the model can be completely dimensionless so that the calculation efficiency and the simulation scale can be further enhanced.

The purpose of this paper is to lay a foundation for the theoretical research of the frequency-regulating feedback and control mechanism of motoneurons, for that the first link of the feedback process is the synaptic inputs of the afferents on motoneurons. The establishment of the model for the postsynaptic responses will facilitate the analysis of synaptic integration [32]. As mentioned above, a motoneuron receives the inputs from the muscle spindle and tendon organ, while the model here only deals with the excitatory input (positive feedback) of the I a afferent of muscle spindle. The tendon organ corresponds to the Ib afferent, which produces inhibitory input (negative feedback). Consequently, in the future studies, in order to investigate the overall dynamic regulation principles of the firing rate of motoneurons and muscle contraction by the stimulus frequency [33-35], the model for the responses of inhibitory synapses needs to be constructed, and the finite element analysis against the whole-cell response should be implemented.

This work was supported by the National Natural Science Foundation of China (61075101), the National Basic Research Program of China (2011CB013203) and the Science and Technology Intercrossing Research Foundation of Shanghai Jiao Tong University (LG2011ZD106).

1 Pecho-Vrieseling E, Sigrist M, Yoshida Y, et al. Specificity of sensory-motor connections encoded by sema3e-plexinD1 recognition. 
Nature, 2009, 459: 842-846

2 Wu S D, Fan X L. Recent advances in the study of structure and function of muscle spindle. Prog Physiol Sci, 2002, 33: 121-125

3 Yin Y H, Guo Z, Chen X, et al. Studies on biomechanics of skeletal muscle based on the working mechanism of myosin motors: An overview. Chin Sci Bull, 2012, 57: 4533-4544

4 Yin Y H, Chen X. Bioelectrochemical control mechanism with variablefrequency regulation for skeletal muscle contraction-Biomechanics of skeletal muscle based on the working mechanism of myosin motors (II). Sci China Tech Sci, 2012, 55: 2115-2125

5 Kernell D. Principles of force gradation in skeletal muscles. Neural Plast, 2003, 10: 69-76

6 Magee J C. Dendritic integration of excitatory synaptic input. Nat Rev Neurosci, 2000, 1: 181-190

7 Kandel E R, Siegelbaum S A. Principles of Neural Science. 4th ed. New York: McGraw-Hill/Appleton and Lange, 2000. 207-228

8 Grillner S. The motor infrastructure: From ion channels to neuronal networks. Nat Rev Neurosci, 2003, 4: 573-586

9 Capaday C, Stein R B. The effects of postsynaptic inhibition on the monosynaptic reflex of the cat at different levels of motoneuron pool activity. Exp Brain Res, 1989, 77: 577-584

10 Pagnotta S E, Lape R, Quitadamo C, et al. Pre- and postsynaptic modulation of glycinergic and gabaergic transmission by muscarinic receptors on rat hypoglossal motoneurons in vitro. Neuroscience, 2005, 130: 783-795

11 Wong A Y C, Graham B P, Billups B, et al. Distinguishing between presynaptic and postsynaptic mechanisms of short-term depression during action potential trains. J Neurosci, 2003, 23: 4868-4877

12 Goldstein S, Rall W. Changes of action potential shape and velocity for changing core conductor geometry. Biophys J, 1974, 14: 731-757

13 Hines M L, Carnevale N T. The NEURON simulation environment. Neural Comput, 1997, 9: 1179-1202

14 Hausser M, Spruston N, Stuart G J. Diversity and dynamics of dendritic signaling. Science, 2000, 290: 739-744

15 Heckman C J, Lee R H, Brownstone R M. Hyperexcitable dendrites in motoneurons and their neuromodulatory control during motor behavior. Trend Neurosci, 2003, 26: 688-695

16 Segev I, London M. Untangling dendrites with quantitative models. Science, 2000, 290: 744-750

17 Rogers J M, McCulloch A D. A collocation-galerkin finite element model of cardiac action potential propagation. IEEE Trans Biomed Eng, 1994, 41: 743-757

18 FitzHugh R. Impulses and physiological states in theoretical models of nerve membrane. Biophys J, 1961, 1: 445-465

19 Destexhe A, Mainen Z F, Sejnowski T J. An efficient method for computing synaptic conductances based on a kinetic model of recep- tor binding. Neural Comput, 1994, 6: 14-18

20 Destexhe A, Mainen Z F. Synthesis of models for excitable membranes, synaptic transmission and neuromodulation using a common kinetic formalism. J Comput Neurosci, 1994, 1: 195-230

21 Lin R C, Scheller R H. Mechanisms of synaptic vesicle exocytosis. Ann Rev Cell Biol, 2000, 16: 19-49

22 Hodgkin A L, Huxley A F. A quantitative description of membrane current and its application to conduction and excitation in nerve. Bull Math Biol, 1990, 52: 25-71

23 Hindmarsh J L, Rose R M. A model of the nerve impulse using two first-order differential equations. Nature, 1982, 296: 162-164

24 Kole M H P, Stuart G J. Signal processing in the axon initial segment. Neuron, 2012, 73: 235-247

25 Colquhoun D, Hawkes A G. On the stochastic properties of single ion channels. Proc Roy Soc Lond B, 1981, 211: 205-235

26 Standley C, Ramsey R L, Usherwood P N R. Gating kinetics of the quisqualate-sensitive glutamate receptor of locust muscle studied using agonist concentration jumps and computer simulations. Biophys $\mathbf{J}$, 1993, 65: 1379-1386

27 Pennartz C M A, Boeijinga P H, Lopes da Silva F H. Contribution of NMDA receptors to postsynaptic potentials and paired-pulse facilitation in identified neurons of the rat nucleus accumbens in vitro. Exp Brain Res, 1991, 86: 190-198

28 Jahr C E, Stevens C F. Voltage dependence of NMDA-activated macroscopic conductances predicted by single-channel kinetics. J Neurosci, 1990, 10: 3178-3182

29 O'Connor J J, Rowan M J, Anwyl R. Tetanically induced LTP involves a similar increase in the AMPA and NMDA receptor components of the excitatory postsynaptic current: Investigations of the involvement of mGlu receptors. J Neurosci, 1995, 15: 2013-2020

30 Markram H, Tsodyks M. Redistribution of synaptic efficacy between neocortical pyramidal neurons. Nature, 1996, 382: 807-810

31 Stuart G, Sakman B. Amplification of EPSPs by axosomatic sodium channels in neocortical pyramidal neurons. Neuron, 1995, 15: 10651076

32 Du J L, Yang X L. Retinal bipolar cells: A model for studying neuronal signal integration. Chin Sci Bull, 1999, 44: 1569-1575

33 Guo Z, Yin Y H. Coupling mechanism of multi-force interactions in the myosin molecular motor. Chin Sci Bull, 2010, 55: 3538-3544

34 Yin Y H, Guo Z. Collective mechanism of molecular motors and a dynamic mechanical model for sarcomere. Sci China Tech Sci, 2011, 54: 2130-2137

35 Guo Z, Yin Y H. A dynamic model of skeletal muscle based on collective behavior of myosin motors-Biomechanics of skeletal muscle based on working mechanism of myosin motors (I). Sci China Tech Sci, 2012, 55: 1589-1595

Open Access This article is distributed under the terms of the Creative Commons Attribution License which permits any use, distribution, and reproduction in any medium, provided the original author(s) and source are credited. 